\title{
CONVOLUTION THEOREMS FOR CLIFFORD FOURIER TRANSFORM AND PROPERTIES
}

\author{
MAWARdi BAHri $^{1}$, RYuichi Ashino ${ }^{2}$, ANd RÉmi VAILlancourT ${ }^{3}$ \\ ${ }^{1}$ Department of Mathematics, Hasanuddin University \\ Tamalanrea Makassar 90245,Indonesia \\ mawardibahri@gmail.com \\ ${ }^{2}$ Division of Mathematical Sciences, Osaka Kyoiku University \\ Osaka 582-8582, Japan \\ ashino@cc.osaka-kyoiku.ac.jp \\ ${ }^{3}$ Department of Mathematics and Statistics, University of Ottawa \\ Ottawa, Ontario, Canada K1N 6N5 \\ remi@uottawa.ca
}

\begin{abstract}
The non-commutativity of the Clifford multiplication gives different aspects from the classical Fourier analysis. We establish main properties of convolution theorems for the Clifford Fourier transform. Some properties of these generalized convolutions are extensions of the corresponding convolution theorems of the classical Fourier transform.

Key words: Clifford convolution, Clifford algebra, Clifford Fourier transform.
\end{abstract}

\begin{abstract}
Abstrak. Tujuan tulisan ini adalah untuk membangun sifat-sifat penting dari teorema konvolusi untuk transformasi Fourier Clifford (TFC). Karena perkalian Clifford adalah tidak komutatif, maka diperoleh sifat-sifat konvolusi dari TFC ini adalah perluasan dari konvolusi dari transformasi Fourier.

Kata kunci: Konvolusi Clifford, aljabar Clifford, transformasi Fourier Clifford.
\end{abstract}

\section{INTRODUCTION}

Recently, several attempts have been made to generalize the classical Fourier transform in the framework of Clifford algebra, so-called the Clifford Fourier transform (CFT). It was first introduced from the mathematical aspect by Brackx et al. $[5,6]$. The CFT was recently used in signal processing $[9,14]$ and in other fields of mathematics and applications. Many generalized transforms, such as the Clifford

2000 Mathematics Subject Classification:15A66, 42B10.

Received: 21-09-2013, revised: 22-04-2014, accepted: 21-05-2014 
wavelet transform, fractional Clifford Fourier transform, and Clifford windowed Fourier transform (see, for example, $[2,7,8,10,12,15]$ ) are closely related to the CFT. One of the most fundamental and important properties of the CFT is the convolution theorem.

Convolution is a mathematical operation with several applications in pure and applied mathematics such as numerical analysis, numerical linear algebra and the design and implementation of finite impulse response filters in signal processing. In $[3,13]$, authors generalized convolution to the quaternion Fourier transform (QFT). They found that the QFT of the real-valued signals are very similar to the classical ones. In this paper, we establish convolution theorems for the CFT. Here we adopt the definition of the CFT suggested by several authors $[1,4,9]$. Because the Clifford multiplication is not commutative, we find important properties of the relationship between the convolution theorems and the CFT. We finally establish the inverse CFT of the product of two CFTs, which is very useful in solving partial differential equations in the Clifford algebra.

This paper is organized as follows. In section 2, we provide some basic knowledge of real Clifford algebra used in the paper. Subsequently, in section 3, we define the CFT and discuss its important properties, which are used to construct the properties of generalized convolution. Next in section 4, we introduce convolution on Clifford algebra $C l_{n, 0}$ and derive its useful properties. Finally, in section 5, we investigate the important properties of the CFT of convolution of Clifford-valued functions and derive the inverse CFT of the product of two CFTs.

\section{Preliminaries}

We shall be working with real Clifford algebras. Let $\left\{\boldsymbol{e}_{1}, \boldsymbol{e}_{2}, \boldsymbol{e}_{3}, \cdots, \boldsymbol{e}_{n}\right\}$ be an orthonormal vector basis of the $n$-dimensional Euclidean vector space $\mathbb{R}^{n}$. The real Clifford algebra over $\mathbb{R}^{n}$, denoted by $C l_{n, 0}$, has the graded $2^{n}$-dimensional basis

$$
\left\{1, e_{1}, e_{2}, \cdots, e_{n}, e_{12}, e_{31}, e_{23}, \cdots, i_{n}=e_{1} e_{2} \cdots e_{n}\right\}
$$

Obviously, for $n=2(\bmod 4)$, the pseudoscalar $i_{n}=\boldsymbol{e}_{1} \boldsymbol{e}_{2} \cdots \boldsymbol{e}_{n}$ anti-commutes with each basis of the Clifford algebra while $i_{n}^{2}=-1$. The noncommutative multiplication of the basis vectors satisfies the rules $\boldsymbol{e}_{i} \boldsymbol{e}_{j}+\boldsymbol{e}_{j} \boldsymbol{e}_{i}=2 \delta_{i j}\left(\delta_{i j}\right.$ denotes the Dirac distribution whose support is $\{i, j\}$ ).

An element of the Clifford algebra is called a multivector and has the following form

$$
f=\sum_{A} e_{A} f_{A}
$$

where $f_{A} \in \mathbb{R}, A \subset\{1,2, \cdots, n\}$. For convenience, we introduce $\langle f\rangle_{k}=\sum_{A} f_{A} \boldsymbol{e}_{A}$ to denote the $k$-vector part of $f(k=0,1,2, \cdots, n)$. Then

$$
f=\sum_{k=0}^{k=n}\langle f\rangle_{k}=\langle f\rangle+\langle f\rangle_{1}+\langle f\rangle_{2}+\cdots+\langle f\rangle_{n},
$$

where $\langle\ldots\rangle=\langle\ldots\rangle_{0}$. 
A multivector $f \in C l_{n, 0}, n=2(\bmod 4)$ can be decomposed as a sum of its even grade part, $f_{\text {even }}$, and its odd grade part, $f_{\text {odd }}$. Thus, we have

$$
f=f_{\text {even }} \oplus f_{\text {odd }},
$$

where

$$
\begin{aligned}
& f_{\text {even }}=\langle f\rangle+\langle f\rangle_{2}+\cdots+\langle f\rangle_{r}, \quad r=2 s, s \in \mathbb{N}, s \leq \frac{n}{2}, \\
& f_{\text {odd }}=\langle f\rangle_{1}+\langle f\rangle_{3}+\cdots+\langle f\rangle_{r}, \quad r=2 s+1, s \in \mathbb{N}, s<\frac{n}{2} .
\end{aligned}
$$

The reverse $\tilde{f}$ of a multivector $f$ is an anti-automorphism given by

$$
\widetilde{f}=\sum_{k=0}^{k=n}(-1)^{k(k-1) / 2}\langle f\rangle_{k},
$$

and hence

$$
\widetilde{f g}=\tilde{g} \tilde{f} \quad \text { for arbitrary } f, g \in C l_{n, 0} .
$$

Decomposition (2) gives the following important proposition (see [4]).

Proposition 2.1. Given a multivector $f \in C l_{n, 0}$ with $n=2(\bmod 4)$. For $\lambda \in \mathbb{R}$ we have

$$
\begin{aligned}
& f e^{i_{n} \lambda}=e^{-i_{n} \lambda} f_{\text {odd }}+e^{i_{n} \lambda} f_{\text {even }}, \\
& \bar{f} e^{i_{n} \lambda}=e^{-i_{n} \lambda} \widetilde{f_{\text {odd }}}+e^{i_{n} \lambda} \widetilde{f_{\text {even }} .}
\end{aligned}
$$

The Clifford product of two vectors splits up into a scalar part (the inner product) and a so-called bivector part (the wedge product):

$$
\boldsymbol{x y}=\boldsymbol{x} \cdot \boldsymbol{y}+\boldsymbol{x} \wedge \boldsymbol{y}
$$

where

$$
\boldsymbol{x} \cdot \boldsymbol{y}=\sum_{i=1}^{n} x_{i} y_{i} \quad \text { and } \quad \boldsymbol{x} \wedge \boldsymbol{y}=\sum_{i=1}^{n} \sum_{j=i+1}^{n} \boldsymbol{e}_{i} \boldsymbol{e}_{j}\left(x_{i} y_{j}-x_{j} y_{i}\right) .
$$

Observe that the square of a vector $\boldsymbol{x}$ is scalar-valued and $\boldsymbol{x}^{2}=\boldsymbol{x} \cdot \boldsymbol{x}+\boldsymbol{x} \wedge \boldsymbol{x}=|\boldsymbol{x}|^{2}$.

We introduce a first order vector differential operator by

$$
\partial_{\boldsymbol{x}}=\sum_{i=1}^{m} \partial_{x_{i}} \boldsymbol{e}_{i}
$$

This operator is the so-called Dirac operator, which may be looked upon as the square root of the Laplacian operator in $\mathbb{R}^{n}: \triangle_{n}=\partial_{x}^{2}$.

Let us consider $L^{2}\left(\mathbb{R}^{n} ; C l_{n, 0}\right)$ as a left module. For $f, g \in L^{2}\left(\mathbb{R}^{n} ; C l_{n, 0}\right)$, an inner product is defined by

$$
\begin{aligned}
(f, g)_{L^{2}\left(\mathbb{R}^{n} ; C l_{n, 0}\right)} & =\int_{\mathbb{R}^{n}} f(\boldsymbol{x}) \widetilde{g(\boldsymbol{x})} d^{n} \boldsymbol{x} \\
& =\sum_{A, B} \boldsymbol{e}_{A} \widetilde{\boldsymbol{e}_{B}} \int_{\mathbb{R}^{n}} f_{A}(\boldsymbol{x}) g_{B}(\boldsymbol{x}) d^{n} \boldsymbol{x} .
\end{aligned}
$$


In particular, if $f=g$, then the scalar part of the above inner product gives the $L^{2}$-norm

$$
\|f\|_{L^{2}\left(\mathbb{R}^{n} ; C l_{n, 0}\right)}^{2}=\int_{\mathbb{R}^{n}} \sum_{A} f_{A}^{2}(\boldsymbol{x}) d^{n} \boldsymbol{x} .
$$

Hereinafter, if not otherwise stated, $n$ is assumed to be $n=2(\bmod 4)$.

\section{Clifford Fourier Transform (CFT)}

3.1. Fundamental Operators. Before we define the CFT, we need to introduce some notation, which will be used in the next section. For $f \in L^{2}\left(\mathbb{R}^{n} ; C l_{n, 0}\right)$, we define the translation and modulation as follows:

$$
\tau_{\boldsymbol{a}} f(\boldsymbol{x})=f(\boldsymbol{x}-\boldsymbol{a}), \quad M_{\boldsymbol{\omega}_{0}} f(\boldsymbol{x})=e^{i_{n} \boldsymbol{\omega}_{0} \cdot \boldsymbol{x}} f(\boldsymbol{x}),
$$

and their composition, which is called the time-frequency shift,

$$
M_{\boldsymbol{\omega}_{0}} \tau_{\boldsymbol{a}} f(\boldsymbol{x})=e^{i_{n} \boldsymbol{\omega}_{0} \cdot \boldsymbol{x}} f(\boldsymbol{x}-\boldsymbol{a}), \quad \boldsymbol{a}, \boldsymbol{\omega}_{0} \in \mathbb{R}^{n} .
$$

Just as in the classical case, we obtain the canonical commutation relations

$$
\tau_{\boldsymbol{a}} M_{\boldsymbol{\omega}_{0}} f=e^{-i_{n} \boldsymbol{\omega}_{0} \cdot \boldsymbol{a}} M_{\boldsymbol{\omega}_{0}} \tau_{\boldsymbol{a}} f .
$$

The following lemma describes the behavior of translation, modulation, and time-frequency shift in the Clifford algebra $C l_{n, 0}$.

Lemma 3.1. If $\boldsymbol{a}, \boldsymbol{\omega}_{0} \in \mathbb{R}^{n}$ and $f, g \in L^{2}\left(\mathbb{R}^{n} ; C l_{n, 0}\right)$, then we have the following:

(i) $\left(f, \tau_{\boldsymbol{a}} g\right)_{L^{2}\left(\mathbb{R}^{n} ; C l_{n, 0}\right)}=\left(\tau_{-\boldsymbol{a}} f, g\right)_{L^{2}\left(\mathbb{R}^{n} ; C l_{n, 0}\right)}$.

(ii) For $g \in L^{2}\left(\mathbb{R}^{n} ; C l_{n, 0}\right), n=3(\bmod 4)$, we obtain

$$
\left(f, M_{\boldsymbol{\omega}_{0}} g\right)_{L^{2}\left(\mathbb{R}^{n} ; C l_{n, 0}\right)}=\left(M_{\boldsymbol{\omega}_{0}} f_{\text {odd }}+M_{-\boldsymbol{\omega}_{0}} f_{\text {even }}, g\right)_{L^{2}\left(\mathbb{R}^{n} ; C l_{n, 0}\right)} .
$$

(iii) For $f \in L^{2}\left(\mathbb{R}^{n} ; C l_{n, 0}\right), n=3(\bmod 4)$, we get

$$
\left(M_{\boldsymbol{\omega}_{0}} f, g\right)_{L^{2}\left(\mathbb{R}^{n} ; C l_{n, 0}\right)}=\left(f, M_{\boldsymbol{\omega}_{0}} g_{\text {odd }}+M_{-\boldsymbol{\omega}_{0}} g_{\text {even }}\right)_{L^{2}\left(\mathbb{R}^{n} ; C l_{n, 0}\right)} .
$$

(iv) Under the assumption stated in (iii), we obtain

$$
\left(M_{\boldsymbol{\omega}_{0}} \tau_{\boldsymbol{a}} f, g\right)_{L^{2}\left(\mathbb{R}^{n} ; C l_{n, 0}\right)}=\left(f, \tau_{-\boldsymbol{a}} M_{\boldsymbol{\omega}_{0}} g_{\mathrm{odd}}+\tau_{-\boldsymbol{a}} M_{-\boldsymbol{\omega}_{0}} g_{\mathrm{even}}\right)_{L^{2}\left(\mathbb{R}^{n} ; C l_{n, 0}\right)} .
$$

Proof. Proof of (i). It follows from (7) that

$$
\begin{aligned}
\left(f, \tau_{\boldsymbol{a}} g\right)_{L^{2}\left(\mathbb{R}^{n} ; C l_{n, 0}\right)} & \left.=\int_{\mathbb{R}^{n}} f(\boldsymbol{x}) g \widetilde{(\boldsymbol{x}-\boldsymbol{a}}\right) d^{n} \boldsymbol{x} \\
& =\int_{\mathbb{R}^{n}} f(\boldsymbol{x}+\boldsymbol{a}) \widetilde{g(\boldsymbol{x})} d^{n} \boldsymbol{x} \\
& =\int_{\mathbb{R}^{n}} \tau_{-\boldsymbol{a}} f(\boldsymbol{x}) \widetilde{g(\boldsymbol{x})} d^{n} \boldsymbol{x} .
\end{aligned}
$$

Proof of (ii). By equations (7) and (9), we easily obtain

$$
\left(f, M_{\boldsymbol{\omega}_{0}} g\right)_{L^{2}\left(\mathbb{R}^{n} ; C l_{n, 0}\right)}=\int_{\mathbb{R}^{n}} f(\boldsymbol{x})\left\{e^{i_{n} \boldsymbol{\omega}_{0} \cdot \boldsymbol{x}} g(\boldsymbol{x})\right\}^{\sim} d^{n} \boldsymbol{x}
$$




$$
\begin{aligned}
& =\int_{\mathbb{R}^{n}} f(\boldsymbol{x})\left\{g(\boldsymbol{x}) e^{i_{n} \boldsymbol{\omega}_{0} \cdot \boldsymbol{x}}\right\}^{\sim} d^{n} \boldsymbol{x} \\
& =\int_{\mathbb{R}^{n}} f(\boldsymbol{x}) e^{-i_{n} \boldsymbol{\omega}_{0} \cdot \boldsymbol{x}} \widetilde{g(\boldsymbol{x})} d^{n} \boldsymbol{x} \\
& =\int_{\mathbb{R}^{n}}\left(f_{\text {odd }}(\boldsymbol{x})+f_{\text {even }}(\boldsymbol{x})\right) e^{-i_{n} \boldsymbol{\omega}_{0} \cdot \boldsymbol{x}} \widetilde{g(\boldsymbol{x})} d^{n} \boldsymbol{x} \\
& =\int_{\mathbb{R}^{n}}\left(e^{i_{n} \boldsymbol{\omega}_{0} \cdot \boldsymbol{x}} f_{\text {odd }}(\boldsymbol{x})+e^{-i_{n} \boldsymbol{\omega}_{0} \cdot \boldsymbol{x}} f_{\text {even }}(\boldsymbol{x})\right) \widetilde{g(\boldsymbol{x})} d^{n} \boldsymbol{x} \\
& =\int_{\mathbb{R}^{n}}\left(M_{\boldsymbol{\omega}_{0}} f_{\text {odd }}(\boldsymbol{x})+M_{-\boldsymbol{\omega}_{0}} f_{\text {even }}(\boldsymbol{x})\right) \widetilde{g(\boldsymbol{x})} d^{n} \boldsymbol{x} .
\end{aligned}
$$

Here, in the second equality of (12), we have used the assumption that ensures to interchange the position.

Proof of (iii). The proof is similar to (ii) and is left to the reader.

Proof of (iv). By simple computations, we get

$$
\begin{aligned}
& \left(M_{\boldsymbol{\omega}_{0}} \tau_{\boldsymbol{a}} f, g\right)_{L^{2}\left(\mathbb{R}^{n} ; C l_{n, 0}\right)} \\
& =\int_{\mathbb{R}^{n}} e^{i_{n} \boldsymbol{\omega}_{0} \cdot \boldsymbol{x}} f(\boldsymbol{x}-\boldsymbol{a}) \widetilde{g(\boldsymbol{x})} d^{n} \boldsymbol{x} \\
& \left.=\int_{\mathbb{R}^{n}} f(\boldsymbol{y})\left(e^{i_{n} \boldsymbol{\omega}_{0} \cdot(\boldsymbol{y}+\boldsymbol{a})} \widetilde{\operatorname{godd}_{\mathrm{od}}}(\boldsymbol{y}+\boldsymbol{a})+e^{i_{n} \boldsymbol{\omega}_{0} \cdot(\boldsymbol{a}+\boldsymbol{y}}\right) \widetilde{g_{\mathrm{even}}}(\boldsymbol{y}+\boldsymbol{a})\right) d^{n} \boldsymbol{y} \\
& =\int_{\mathbb{R}^{n}} f(\boldsymbol{y})\left\{g_{\text {odd }}(\boldsymbol{y}+\boldsymbol{a}) e^{-i_{n} \boldsymbol{\omega}_{0} \cdot(\boldsymbol{y}+\boldsymbol{a}}\right\}^{\sim} d^{n} \boldsymbol{y} \\
& +\int_{\mathbb{R}^{n}} f(\boldsymbol{y})\left\{g_{\text {even }}(\boldsymbol{y}+\boldsymbol{a}) e^{-i_{n} \boldsymbol{\omega}_{0} \cdot(\boldsymbol{y}+\boldsymbol{a})}\right\} \sim d^{n} \boldsymbol{y} \\
& =\int_{\mathbb{R}^{n}} f(\boldsymbol{y})\left\{e^{i_{n} \boldsymbol{\omega}_{0} \cdot(\boldsymbol{y}+\boldsymbol{a})} g_{\text {odd }}(\boldsymbol{y}+\boldsymbol{a})\right\}^{\sim} d^{n} \boldsymbol{y} \\
& +\int_{\mathbb{R}^{n}} f(\boldsymbol{y})\left\{e^{-i_{n} \boldsymbol{\omega}_{0} \cdot(\boldsymbol{y}+\boldsymbol{a})} g_{\mathrm{even}}(\boldsymbol{y}+\boldsymbol{a})\right\}^{\sim} d^{n} \boldsymbol{y} \\
& =\int_{\mathbb{R}^{n}} f(\boldsymbol{y})\left(\left\{\tau_{-\boldsymbol{a}} M_{\boldsymbol{\omega}_{0}} g_{\text {odd }}(\boldsymbol{y})\right\}^{\sim}+\left\{\tau_{-\boldsymbol{a}} M_{-\boldsymbol{\omega}_{0}} g_{\mathrm{even}}(\boldsymbol{y})\right\}^{\sim}\right) d^{n} \boldsymbol{y} .
\end{aligned}
$$

Here, in the second and third equalities of (13), we have used the assumption and properties of the decomposition of multivector $g$.

3.2. Definition of CFT. The $C l_{n, 0}$ Clifford Fourier transform (CFT) is a generalization of the FT in Clifford algebra obtained by replacing the FT kernel with the Clifford Fourier kernel. For detailed discussions of the properties of the CFT and their proofs, see, e.g., [4, 9].

Definition 3.2. The $C F T$ of $f \in L^{2}\left(\mathbb{R}^{n} ; C l_{n, 0}\right)$ is the function $\mathcal{F}\{f\} \in L^{2}\left(\mathbb{R}^{n} ; C l_{n, 0}\right)$ given by

$$
\mathcal{F}\{f\}(\boldsymbol{\omega})=\hat{f}(\boldsymbol{\omega})=\int_{\mathbb{R}^{n}} f(\boldsymbol{x}) e^{-i_{n} \boldsymbol{\omega} \cdot \boldsymbol{x}} d^{n} \boldsymbol{x},
$$

with $\boldsymbol{x}, \boldsymbol{\omega} \in \mathbb{R}^{n}$. 
Decomposing the multivector $f$ into $f_{\text {even }}$ and $f_{\text {odd }}$, equation (14) can be rewritten as

$$
\mathcal{F}\{f\}(\boldsymbol{\omega})=\int_{\mathbb{R}^{n}} e^{i_{n} \boldsymbol{\omega} \cdot \boldsymbol{x}} f_{\text {odd }}(\boldsymbol{x}) d^{n} \boldsymbol{x}+\int_{\mathbb{R}^{n}} e^{-i_{n} \boldsymbol{\omega} \cdot \boldsymbol{x}} f_{\text {even }}(\boldsymbol{x}) d^{n} \boldsymbol{x} .
$$

The Clifford exponential $e^{-i_{n} \boldsymbol{\omega} \cdot \boldsymbol{x}}$ is often called the Clifford Fourier kernel. For dimension $n=3(\bmod 4)$, this kernel commutes with all elements of the Clifford algebra $C l_{n, 0}$, but for $n=2(\bmod 4)$ it does not. Notice that the different commutation rules of the pseudoscalar $i_{n}$ play a crucial rule in establishing the properties of the convolution theorems of the CFT.

In the following, we collect the fundamental properties of the CFT.

Lemma 3.3. If $f \in L^{2}\left(\mathbb{R}^{n} ; C l_{n, 0}\right)$, then the following results hold:

$$
\begin{aligned}
\mathcal{F}\left\{\tau_{\boldsymbol{a}} f\right\}(\boldsymbol{\omega})= & \mathcal{F}\{f\}(\boldsymbol{\omega}) e^{-i_{n} \boldsymbol{\omega} \cdot \boldsymbol{a}} \\
= & e^{i_{n} \boldsymbol{\omega} \cdot \boldsymbol{a}} \mathcal{F}\left\{f_{\text {odd }}\right\}(\boldsymbol{\omega})+e^{-i_{n} \boldsymbol{\omega} \cdot \boldsymbol{a}} \mathcal{F}\left\{f_{\text {even }}\right\}(\boldsymbol{\omega}) . \\
\mathcal{F}\left\{M_{\boldsymbol{\omega}_{0}} f\right\}(\boldsymbol{\omega})= & \mathcal{F}\left\{f_{\text {odd }}\right\}\left(\boldsymbol{\omega}_{0}-\boldsymbol{\omega}\right)+\mathcal{F}\left\{f_{\text {even }}\right\}\left(\boldsymbol{\omega}+\boldsymbol{\omega}_{0}\right) . \\
\mathcal{F}\left\{M_{\boldsymbol{\omega}_{0}} \tau_{\boldsymbol{a}} f\right\}(\boldsymbol{\omega})= & e^{-i_{n}\left(\boldsymbol{\omega}_{0}-\boldsymbol{\omega}\right) \cdot \boldsymbol{a}} \mathcal{F}\left\{f_{\text {odd }}\right\}\left(\boldsymbol{\omega}_{0}-\boldsymbol{\omega}\right) \\
& +e^{-i_{n}\left(\boldsymbol{\omega}_{0}+\boldsymbol{\omega}\right) \cdot \boldsymbol{a}} \mathcal{F}\left\{f_{\text {even }}\right\}\left(\boldsymbol{\omega}+\boldsymbol{\omega}_{0}\right) .
\end{aligned}
$$

Theorem 3.4. Suppose that $\mathcal{F}\{f\} \in L^{2}\left(\mathbb{R}^{n} ; C l_{n, 0}\right)$. Then $\mathcal{F}\{f\}$ is invertible and its inverse is calculated by the formula

$$
\mathcal{F}^{-1}[\mathcal{F}\{f\}(\boldsymbol{\omega})](\boldsymbol{x})=f(\boldsymbol{x})=\frac{1}{(2 \pi)^{n}} \int_{\mathbb{R}^{n}} \mathcal{F}\{f\}(\boldsymbol{\omega}) e^{i_{n} \boldsymbol{\omega} \cdot \boldsymbol{x}} d^{n} \boldsymbol{\omega} .
$$

Proof. Substituting (14) into (16) yields

$$
\begin{aligned}
\mathcal{F}^{-1}[\mathcal{F}\{f\} \boldsymbol{\omega}](\boldsymbol{x}) & =\frac{1}{(2 \pi)^{n}} \int_{\mathbb{R}^{n}} \int_{\mathbb{R}^{n}} f(\boldsymbol{y}) e^{-i_{n} \boldsymbol{\omega} \cdot \boldsymbol{y}} d^{n} \boldsymbol{y} e^{i_{n} \boldsymbol{\omega} \cdot \boldsymbol{x}} d^{n} \boldsymbol{\omega} \\
& =\int_{\mathbb{R}^{n}} f(\boldsymbol{y}) \frac{1}{(2 \pi)^{n}} \int_{\mathbb{R}^{n}} e^{i_{n}(\boldsymbol{x}-\boldsymbol{y}) \cdot \boldsymbol{\omega}} d^{n} \boldsymbol{\omega} d^{n} \boldsymbol{y} \\
& =\int_{\mathbb{R}^{n}} f(\boldsymbol{y}) \delta(\boldsymbol{x}-\boldsymbol{y}) d^{n} \boldsymbol{y} \\
& =f(\boldsymbol{x}) .
\end{aligned}
$$

Equation (16) is called the Clifford Fourier integral theorem. It describes how to get from the transform $\mathcal{F}\{f\}$ back to the original function $f$.

It is straightforward to see that the inverse CFT and the CFT share the same properties. One may check the properties of the inverse CFT analogous to those in Lemma 3.3. For an example,

$$
\begin{aligned}
\mathcal{F}^{-1} & {\left[\mathcal{F}\left\{\tau_{\boldsymbol{a}} f\right\}(\boldsymbol{\omega})\right](\boldsymbol{x}) } \\
& =\frac{1}{(2 \pi)^{n}} \int_{\mathbb{R}^{n}} \mathcal{F}\left\{\tau_{\boldsymbol{a}} f\right\}(\boldsymbol{\omega}) e^{i_{n} \boldsymbol{\omega} \cdot \boldsymbol{x}} d^{n} \boldsymbol{\omega}
\end{aligned}
$$




$$
\begin{aligned}
= & \frac{1}{(2 \pi)^{n}} \int_{\mathbb{R}^{n}}\left(e^{i_{n} \boldsymbol{\omega} \cdot \boldsymbol{a}} \mathcal{F}\left\{f_{\text {odd }}\right\}(\boldsymbol{\omega})+e^{-i_{n} \boldsymbol{\omega} \cdot \boldsymbol{a}} \mathcal{F}\left\{f_{\text {even }}\right\}(\boldsymbol{\omega})\right) e^{i_{n} \boldsymbol{\omega} \cdot \boldsymbol{x}} d^{n} \boldsymbol{\omega} \\
= & \frac{1}{(2 \pi)^{n}} \int_{\mathbb{R}^{n}} e^{i_{n} \boldsymbol{\omega} \cdot \boldsymbol{a}} \mathcal{F}\left\{f_{\text {odd }}\right\}(\boldsymbol{\omega}) e^{i_{n} \boldsymbol{\omega} \cdot \boldsymbol{x}} d^{n} \boldsymbol{\omega} \\
& +\frac{1}{(2 \pi)^{n}} \int_{\mathbb{R}^{n}} e^{-i_{n} \boldsymbol{\omega} \cdot \boldsymbol{a}} \mathcal{F}\left\{f_{\text {even }}\right\}(\boldsymbol{\omega}) e^{i_{n} \boldsymbol{\omega} \cdot \boldsymbol{x}} d^{n} \boldsymbol{\omega} \\
= & e^{i_{n} \boldsymbol{\omega} \cdot \boldsymbol{a}} \mathcal{F}^{-1}\left[\mathcal{F}\left\{f_{\text {odd }}\right\}(\boldsymbol{\omega})\right](\boldsymbol{x})+e^{-i_{n} \boldsymbol{\omega} \cdot \boldsymbol{a}} \mathcal{F}^{-1}\left[\mathcal{F}\left\{f_{\text {even }}\right\}(\boldsymbol{\omega})\right](\boldsymbol{x}) .
\end{aligned}
$$

\section{Clifford Convolution And Its Properties}

In this section, we introduce the Clifford convolution and establish its important properties. Ebling and Scheuermann [9] distinguish between right and left Clifford convolutions due to the non-commutative property of the Clifford multiplication. Here, we only consider one kind of Clifford convolution. Let us first define the convolution of two Clifford-valued functions.

Definition 4.1. The Clifford convolution $f \star g$ of $f$ and $g$ belong to $L^{2}\left(\mathbb{R}^{n} ; C l_{n, 0}\right)$ is defined by

$$
\begin{aligned}
(f \star g)(\boldsymbol{x}) & =\int_{\mathbb{R}^{n}} f(\boldsymbol{y}) g(\boldsymbol{x}-\boldsymbol{y}) d^{n} \boldsymbol{y} \\
& =\int_{\mathbb{R}^{n}} \sum_{A, B} \boldsymbol{e}_{A} \boldsymbol{e}_{B} f_{A}(\boldsymbol{y}) g_{B}(\boldsymbol{x}-\boldsymbol{y}) d^{n} \boldsymbol{y} .
\end{aligned}
$$

Since, in general, the basis vectors $\boldsymbol{e}_{A} \boldsymbol{e}_{B} \neq \boldsymbol{e}_{B} \boldsymbol{e}_{A}$, the Clifford convolution is not commutative, i.e., $(f \star g) \neq(g \star f)$. It is clear that the Clifford convolution of $f$ and $g$ is a binary operation, which combines shifting, geometric product and integration.

If we perform the change of variables $\boldsymbol{z}=\boldsymbol{x}-\boldsymbol{y}$ and relabel $\boldsymbol{z}$ back to $\boldsymbol{y}$, then equation (17) can be written as

$$
(f \star g)(\boldsymbol{x})=\int_{\mathbb{R}^{n}} f(\boldsymbol{x}-\boldsymbol{y}) g(\boldsymbol{y}) d^{n} \boldsymbol{y} .
$$

Lemma 4.2 (Linearity). Let $f, g, h \in L^{2}\left(\mathbb{R}^{n} ; C l_{n, 0}\right)$ and $\alpha, \beta \in C l_{n, 0}$. Then, we have

$$
\begin{aligned}
& (\alpha f+\beta g) \star h=\alpha(f \star h)+\beta(g \star h) . \\
& h \star(f \alpha+g \beta)=(h \star f) \alpha+(h \star g) \beta .
\end{aligned}
$$

Lemma 4.3 (Shifting). Let $f \in L^{2}\left(\mathbb{R}^{n} ; C l_{n, 0}\right)$. Then we have

$$
\begin{aligned}
\tau_{\boldsymbol{a}}(f \star g)(\boldsymbol{x}) & =\left(f \star \tau_{\boldsymbol{a}} g\right)(\boldsymbol{x})=\left(\tau_{\boldsymbol{a}} f \star g\right)(\boldsymbol{x}), \\
\tau_{\boldsymbol{a}}(g \star f)(\boldsymbol{x}) & =\left(g \star \tau_{\boldsymbol{a}} f\right)(\boldsymbol{x})=\left(\tau_{\boldsymbol{a}} g \star f\right)(\boldsymbol{x}), \quad \boldsymbol{a} \in \mathbb{R}^{n} .
\end{aligned}
$$


Proof. We only prove (20), the proof of (21) being similar. A direct calculation yields

$$
\begin{aligned}
\tau_{\boldsymbol{a}}(f \star g)(\boldsymbol{x}) & =\int_{\mathbb{R}^{n}} f(\boldsymbol{y}) g(\boldsymbol{x}-\boldsymbol{y}-\boldsymbol{a}) d^{n} \boldsymbol{y} \\
& =\int_{\mathbb{R}^{n}} f(\boldsymbol{y}) \tau_{\boldsymbol{a}} g(\boldsymbol{x}-\boldsymbol{y}) d^{n} \boldsymbol{y} \\
& =\left(f \star \tau_{\boldsymbol{a}} g\right)(\boldsymbol{x}) .
\end{aligned}
$$

On the other hand, by the change of variables, $\boldsymbol{z}=\boldsymbol{x}-\boldsymbol{y}-\boldsymbol{a}$, we easily get

$$
\begin{aligned}
\tau_{\boldsymbol{a}}(f \star g)(\boldsymbol{x}) & =\int_{\mathbb{R}^{n}} f(\boldsymbol{x}-\boldsymbol{z}-\boldsymbol{a}) g(\boldsymbol{z}) d^{n} \boldsymbol{z} \\
& =\int_{\mathbb{R}^{n}} \tau_{\boldsymbol{a}} f(\boldsymbol{x}-\boldsymbol{z}) g(\boldsymbol{z}) d^{n} \boldsymbol{z} \\
& =\left(\tau_{\boldsymbol{a}} f \star g\right)(\boldsymbol{x}) .
\end{aligned}
$$

This completes the proof.

Equations (20) and (21) tell us that the Clifford convolutions commute with translations.

Remark 4.4. Due to the noncommutativity of the Clifford convolution, it is easy to see that $\left(f \star \tau_{\boldsymbol{a}} g\right)(\boldsymbol{x}) \neq\left(\tau_{\boldsymbol{a}} g \star f\right)$ and $\left(\tau_{\boldsymbol{a}} f \star g\right)(\boldsymbol{x}) \neq\left(g \star \tau_{\boldsymbol{a}} f\right)$ in general.

Lemma 4.5 (Reversion). Let $f, g \in L^{2}\left(\mathbb{R}^{n} ; C l_{n, 0}\right)$. Then, we have

$$
\widetilde{(f \star g)}(\boldsymbol{x})=(\tilde{g} \star \tilde{f})(\boldsymbol{x}) \text {. }
$$

Proof. A straightforward computation gives

$$
\begin{aligned}
\widetilde{(f \star g)}(\boldsymbol{x}) & =\int_{\mathbb{R}^{n}}\{f(\boldsymbol{y}) g(\boldsymbol{x}-\boldsymbol{y})\}^{\sim} d^{n} \boldsymbol{y} \\
& \stackrel{(4)}{=} \int_{\mathbb{R}^{n}} \tilde{g}(\boldsymbol{x}-\boldsymbol{y}) \tilde{f}(\boldsymbol{y}) d^{n} \boldsymbol{y} \\
& =\int_{\mathbb{R}^{n}} \tilde{g}(\boldsymbol{z}) \tilde{f}(\boldsymbol{x}-\boldsymbol{z}) d^{n} \boldsymbol{z} \\
& =(\tilde{g} \star \tilde{f})(\boldsymbol{x}),
\end{aligned}
$$

which was to be proved.

\section{Main Results}

In this section, we investigate some important properties of the CFT of convolution of two Clifford-valued functions. We find that most of these properties are extensions of the classical case. The following theorem gives the relationship between the reversion of Clifford convolution and its CFT. 
Theorem 5.1. Let $f, g \in L^{2}\left(\mathbb{R}^{n} ; C l_{n, 0}\right)$. Denote by $g_{\text {odd }}$ (resp. $\left.g_{\text {even }}\right)$, the odd (resp. even) grade part of $g$. Then

$$
\begin{aligned}
& \left.\mathcal{F}\{\widetilde{f \star g}\}(\boldsymbol{\omega})=\left(\widetilde{\mathcal{F}\left\{g_{\text {odd }}\right.}\right\}(-\boldsymbol{\omega})+\widetilde{\mathcal{F}\left\{g_{\text {even }}\right\}}(\boldsymbol{\omega})\right) \mathcal{F}\left\{\widetilde{\left.f_{\text {odd }}\right\}}(\boldsymbol{\omega})\right. \\
& \left.+\widetilde{\mathcal{F}\left\{g_{\text {odd }}\right\}}(\boldsymbol{\omega})+\widetilde{\mathcal{F}\left\{g_{\text {even }}\right\}}(-\boldsymbol{\omega})\right) \mathcal{F}\left\{\widehat{\left.f_{\text {even }}\right\}}(-\boldsymbol{\omega}) .\right.
\end{aligned}
$$

Proof. An application of the CFT definition combined with the Clifford convolution property of Lemma 4.5 gives

$$
\begin{aligned}
\mathcal{F}\{\widetilde{f \star g}\}(\boldsymbol{\omega}) & =\mathcal{F}\{\tilde{g} \star \tilde{f}\}(\boldsymbol{\omega}) \\
& \stackrel{(14)}{=} \int_{\mathbb{R}^{n}}(\tilde{g} \star \tilde{f}) e^{-i_{n} \boldsymbol{\omega} \cdot \boldsymbol{x}} d^{n} \boldsymbol{x} \\
& \stackrel{(17)}{=} \int_{\mathbb{R}^{n}} \int_{\mathbb{R}^{n}} \tilde{g}(\boldsymbol{y}) \widetilde{f(\boldsymbol{x}-\boldsymbol{y})} d^{n} \boldsymbol{y} e^{-i_{n} \boldsymbol{\omega} \cdot \boldsymbol{x}} d^{n} \boldsymbol{x} \\
& =\int_{\mathbb{R}^{n}} \tilde{g}(\boldsymbol{y})\left[\int_{\mathbb{R}^{n}} \widetilde{f(\boldsymbol{x}-\boldsymbol{y})} e^{-i_{n} \boldsymbol{\omega} \cdot \boldsymbol{x}} d^{n} \boldsymbol{x}\right] d^{n} \boldsymbol{y} \\
& =\int_{\mathbb{R}^{n}} \tilde{g}(\boldsymbol{y})\left[\int_{\mathbb{R}^{n}} \widetilde{f(\boldsymbol{z})} e^{-i_{n} \boldsymbol{\omega} \cdot(\boldsymbol{y}+\boldsymbol{z})} d^{n} \boldsymbol{z}\right] d^{n} \boldsymbol{y},
\end{aligned}
$$

where the last equality follows from the change of variables $\boldsymbol{z}=\boldsymbol{x}-\boldsymbol{y}$. By splitting $f$ into its even grade and odd grade parts, the above identity may be rewritten as

$$
\begin{aligned}
& \mathcal{F}\{\widetilde{f \star g}\}(\boldsymbol{\omega}) \\
& =\int_{\mathbb{R}^{n}} \tilde{g}(\boldsymbol{y})\left[\int_{\mathbb{R}^{n}} e^{i_{n} \boldsymbol{\omega} \cdot \boldsymbol{y}}\left\{e^{i_{n} \boldsymbol{\omega} \cdot \boldsymbol{z}} f_{\mathrm{odd}}(\boldsymbol{z})\right\}^{\sim} d^{n} \boldsymbol{z}\right. \\
& \left.+\int_{\mathbb{R}^{n}} e^{-i_{n} \boldsymbol{\omega} \cdot \boldsymbol{y}}\left\{e^{i_{n} \boldsymbol{\omega} \cdot \boldsymbol{z}} f_{\text {even }}(\boldsymbol{z})\right\}^{\sim} d^{n} \boldsymbol{z}\right] d^{n} \boldsymbol{y} \\
& =\int_{\mathbb{R}^{n}} \tilde{g}(\boldsymbol{y})\left[\int_{\mathbb{R}^{n}} e^{i_{n} \boldsymbol{\omega} \cdot \boldsymbol{y}}\left\{f_{\text {odd }}(\boldsymbol{z}) e^{-i_{n} \boldsymbol{\omega} \cdot \boldsymbol{z}}\right\} \sim d^{n} \boldsymbol{z}\right. \\
& \left.+\int_{\mathbb{R}^{n}} e^{-i_{n} \boldsymbol{\omega} \cdot \boldsymbol{y}}\left\{f_{\text {even }}(\boldsymbol{z}) e^{i_{n} \boldsymbol{\omega} \cdot \boldsymbol{z}}\right\} \sim d^{n} \boldsymbol{z}\right] d^{n} \boldsymbol{y} \\
& =\int_{\mathbb{R}^{n}} \tilde{g}(\boldsymbol{y}) e^{i_{n} \boldsymbol{\omega} \cdot \boldsymbol{y}} d^{n} \boldsymbol{y} \mathcal{F}\left\{\widehat{\left.f_{\text {odd }}\right\}}(\boldsymbol{\omega})+\int_{\mathbb{R}^{n}} \tilde{g}(\boldsymbol{y}) e^{-i_{n} \boldsymbol{\omega} \cdot \boldsymbol{y}} d^{n} \boldsymbol{y} \mathcal{F}\left\{\widetilde{\left.f_{\text {even }}\right\}(-\boldsymbol{\omega})}\right. \text {. }\right.
\end{aligned}
$$

Again we decompose the multivector $g$ into its even grade and odd grade parts to get

$$
\begin{aligned}
& \mathcal{F}\{\widetilde{f \star g}\}(\boldsymbol{\omega}) \\
& =\int_{\mathbb{R}^{n}}\left(\widetilde{g_{\text {odd }}}(\boldsymbol{y})+\widetilde{g_{\text {even }}}(\boldsymbol{y})\right) e^{i_{n} \boldsymbol{\omega} \cdot \boldsymbol{y}} d^{n} \boldsymbol{y} \mathcal{F}\left\{\widetilde{\left.f_{\text {odd }}\right\}}(\boldsymbol{\omega})\right. \\
& +\int_{\mathbb{R}^{n}}\left(\widetilde{g_{\text {odd }}}(\boldsymbol{y})+\widetilde{g_{\text {even }}}(\boldsymbol{y})\right) e^{-i_{n} \boldsymbol{\omega} \cdot \boldsymbol{y}} d^{n} \boldsymbol{y} \mathcal{F}\left\{\widetilde{\left.f_{\text {even }}\right\}}(-\boldsymbol{\omega})\right. \\
& =\left[\int_{\mathbb{R}^{n}}\left\{g_{\text {odd }}(\boldsymbol{y}) e^{i_{n} \boldsymbol{\omega} \cdot \boldsymbol{y}}\right\} \sim d^{n} \boldsymbol{y}+\int_{\mathbb{R}^{n}}\left\{g_{\text {even }}(\boldsymbol{y}) e^{-i_{n} \boldsymbol{\omega} \cdot \boldsymbol{y}}\right\} \sim d^{n} \boldsymbol{y}\right] \mathcal{F}\left\{\widetilde{f_{\text {odd }}}\right\}(\boldsymbol{\omega})
\end{aligned}
$$




$$
\begin{aligned}
& +\left[\int_{\mathbb{R}^{n}}\left\{g_{\text {odd }}(\boldsymbol{y}) e^{-i_{n} \boldsymbol{\omega} \cdot \boldsymbol{y}}\right\}^{\sim} d^{n} \boldsymbol{y}+\int_{\mathbb{R}^{n}}\left\{g_{\text {even }}(\boldsymbol{y}) e^{i_{n} \boldsymbol{\omega} \cdot \boldsymbol{y}}\right\}^{\sim} d^{n} \boldsymbol{y}\right] \mathcal{F}\left\{\widetilde{\left.f_{\text {even }}\right\}(-\boldsymbol{\omega})}\right. \\
= & \left(\widetilde{\mathcal{F}\left\{g_{\text {odd }}\right\}}(-\boldsymbol{\omega})+\widetilde{\mathcal{F}\left\{g_{\text {even }}\right\}}(\boldsymbol{\omega})\right) \mathcal{F}\left\{\widetilde{\left.f_{\text {odd }}\right\}}(\boldsymbol{\omega})\right. \\
& +\left(\widetilde{\mathcal{F}\left\{g_{\text {odd }}\right\}}(\boldsymbol{\omega})+\widetilde{\mathcal{F}\left\{g_{\text {even }}\right\}}(-\boldsymbol{\omega})\right) \mathcal{F}\left\{\widetilde{\left.f_{\text {even }}\right\}}(-\boldsymbol{\omega}) .\right.
\end{aligned}
$$

This concludes the proof.

As an immediate consequence of Theorem 5.1, we get the following corollaries.

Corollary 5.2. Let $g$ be as above. For $f \in L^{2}\left(\mathbb{R}^{n} ; C l_{n, 0}\right)$ with $n=3(\bmod 4)$, equation (23) reduces to

$$
\left.\left.\mathcal{F}\{\widetilde{f \star g}\}(\boldsymbol{\omega})=\widetilde{\mathcal{F}\left\{g_{\text {odd }}\right.}\right\}(\boldsymbol{\omega})+\widetilde{\mathcal{F}\left\{g_{\text {even }}\right\}}(-\boldsymbol{\omega})\right) \mathcal{F}\{\widetilde{f\}(-\boldsymbol{\omega}}) .
$$

Corollary 5.3. If $f, g \in L^{2}\left(\mathbb{R}^{n} ; C l_{n, 0}\right)$ with $n=3(\bmod 4)$, then equation (23) becomes

$$
\mathcal{F}\{\widetilde{f \star g}\}(\boldsymbol{\omega})=\widetilde{\mathcal{F}\{g\}(\boldsymbol{\omega})} \mathcal{F}\left\{\widetilde{\left.f_{\text {odd }}\right\}}(\boldsymbol{\omega})+\widetilde{\mathcal{F}\{g\}(-\boldsymbol{\omega})} \mathcal{F}\left\{\widetilde{\left.f_{\text {even }}\right\}(}-\boldsymbol{\omega}\right) .\right.
$$

Proof. An alternative proof of Corollary 5.2 uses [4, (4.59) of Theorem 4.33], i.e.,

$$
\begin{aligned}
\mathcal{F}\{ & \widetilde{f} \star g\} \\
= & \mathcal{F}\{\tilde{\boldsymbol{\omega}}\}(\boldsymbol{\omega}) \mathcal{F}\{\tilde{f}\}(\boldsymbol{\omega}) \\
= & {\left[\int_{\mathbb{R}^{n}} \tilde{g}(\boldsymbol{y}) e^{-i_{n} \boldsymbol{\omega} \cdot \boldsymbol{y}} d^{n} \boldsymbol{y}\right]\left[\int_{\mathbb{R}^{n}} \tilde{f}(\boldsymbol{x}) e^{-i_{n} \boldsymbol{\omega} \cdot \boldsymbol{x}} d^{n} \boldsymbol{x}\right] } \\
= & {\left[\int_{\mathbb{R}^{n}}\left\{g_{\text {odd }}(\boldsymbol{y}) e^{-i_{n} \boldsymbol{\omega} \cdot \boldsymbol{y}}\right\}^{\sim} d^{n} \boldsymbol{y}+\int_{\mathbb{R}^{n}}\left\{g_{\mathrm{even}}(\boldsymbol{y}) e^{i_{n} \boldsymbol{\omega} \cdot \boldsymbol{y}}\right\}^{\sim} d^{n} \boldsymbol{y}\right] } \\
& \times\left[\int_{\mathbb{R}^{n}}\left\{g(\boldsymbol{x}) e^{i_{n} \boldsymbol{\omega} \cdot \boldsymbol{x}}\right\}^{\sim} d^{n} \boldsymbol{x}\right] \\
= & \left.\left(\widetilde{\mathcal{F}\left\{g_{\text {odd }}\right.}\right\}(\boldsymbol{\omega})+\widetilde{\mathcal{F}\left\{g_{\text {even }}\right\}}(-\boldsymbol{\omega})\right) .
\end{aligned}
$$

It is worth noting here that a similar argument cannot be applied to prove Corollary 5.3. Its proof follows directly from Theorem 5.1.

We next establish the shift property of the convolution theorem of the CFT. The proof of this property uses the shift property of the CFT and the decomposition of a multivector $f$.

Theorem 5.4. Let $f, g \in L^{2}\left(\mathbb{R}^{n} ; C l_{n, 0}\right)$. Then

$$
\begin{aligned}
\mathcal{F}\left\{\tau_{\boldsymbol{a}} f \star g\right\}(\boldsymbol{\omega})=\mathcal{F}\left\{f \star \tau_{\boldsymbol{a}} g\right\}(\boldsymbol{\omega})=\mathcal{F}\{f\} & (-\boldsymbol{\omega}) e^{i_{n} \boldsymbol{\omega} \cdot \boldsymbol{a}} \mathcal{F}\left\{g_{\text {odd }}\right\}(\boldsymbol{\omega}) \\
& +\mathcal{F}\{f\}(\boldsymbol{\omega}) e^{-i_{n} \boldsymbol{\omega} \cdot \boldsymbol{a}} \mathcal{F}\left\{g_{\mathrm{even}}\right\}(\boldsymbol{\omega}) .
\end{aligned}
$$

Proof. By the definition of CFT, we easily get

$$
\begin{aligned}
& \mathcal{F}\left\{\tau_{\boldsymbol{a}} f \star g\right\}(\boldsymbol{\omega}) \\
& \stackrel{(14)}{=} \int_{\mathbb{R}^{n}} \int_{\mathbb{R}^{n}} f(\boldsymbol{y}-\boldsymbol{a}) g(\boldsymbol{x}-\boldsymbol{y}) d^{n} \boldsymbol{y} e^{-i_{n} \boldsymbol{\omega} \cdot \boldsymbol{x}} d^{n} \boldsymbol{x}
\end{aligned}
$$




$$
\begin{aligned}
= & \int_{\mathbb{R}^{n}} f(\boldsymbol{y}-\boldsymbol{a})\left[\int_{\mathbb{R}^{n}} g(\boldsymbol{x}-\boldsymbol{y}) e^{-i_{n} \boldsymbol{\omega} \cdot \boldsymbol{x}} d^{n} \boldsymbol{x}\right] d^{n} \boldsymbol{y} \\
= & \int_{\mathbb{R}^{n}} f(\boldsymbol{y}-\boldsymbol{a})\left[\int_{\mathbb{R}^{n}} g(\boldsymbol{z}) e^{-i_{n} \boldsymbol{\omega} \cdot(\boldsymbol{y}+\boldsymbol{z})} d^{n} \boldsymbol{z}\right] d^{n} \boldsymbol{y} \\
= & \int_{\mathbb{R}^{n}} f(\boldsymbol{y}-\boldsymbol{a})\left[\int_{\mathbb{R}^{n}} e^{i_{n} \boldsymbol{\omega} \cdot \boldsymbol{y}} g_{\mathrm{odd}}(\boldsymbol{z})+\int_{\mathbb{R}^{n}} e^{-i_{n} \boldsymbol{\omega} \cdot \boldsymbol{y}} g_{\mathrm{even}}(\boldsymbol{z})\right] e^{-i_{n} \boldsymbol{\omega} \cdot \boldsymbol{z}} d^{n} \boldsymbol{z} d^{n} \boldsymbol{y} \\
= & \int_{\mathbb{R}^{n}} f(\boldsymbol{y}-\boldsymbol{a}) e^{i_{n} \boldsymbol{\omega} \cdot \boldsymbol{y}} d^{n} \boldsymbol{y} \mathcal{F}\left\{g_{\text {odd }}\right\}(\boldsymbol{\omega}) \\
& +\int_{\mathbb{R}^{n}} f(\boldsymbol{y}-\boldsymbol{a}) e^{-i_{n} \boldsymbol{\omega} \cdot \boldsymbol{y}} d^{n} \boldsymbol{y} \mathcal{F}\left\{g_{\mathrm{even}}\right\}(\boldsymbol{\omega}) \\
= & \mathcal{F}\{f\}(-\boldsymbol{\omega}) e^{i_{n} \boldsymbol{\omega} \cdot \boldsymbol{a}} \mathcal{F}\left\{g_{\mathrm{odd}}\right\}(\boldsymbol{\omega})+\mathcal{F}\{f\}(\boldsymbol{\omega}) e^{-i_{n} \boldsymbol{\omega} \cdot \boldsymbol{a}} \mathcal{F}\left\{g_{\text {even }}\right\}(\boldsymbol{\omega}) .
\end{aligned}
$$

For the third equality, we have used the substitution of variable $\boldsymbol{z}=\boldsymbol{x}-\boldsymbol{y}$. For the last equality, we have used the shift property of the CFT. This completes the proof of (26).

We have the following simple corollary to Theorem 5.4.

Corollary 5.5. When $g \in L^{2}\left(\mathbb{R}^{n} ; C l_{n, 0}\right)$ with $n=3(\bmod 4)$, Theorem 5.4 takes the form

$$
\mathcal{F}\left\{\tau_{\boldsymbol{a}} f \star g\right\}(\boldsymbol{\omega})=\mathcal{F}\{f\}(\boldsymbol{\omega}) e^{-i_{n} \boldsymbol{\omega} \cdot \boldsymbol{a}} \mathcal{F}\{g\}(\boldsymbol{\omega}) .
$$

Or, equivalently,

$$
\mathcal{F}\left\{\tau_{\boldsymbol{a}} f \star g\right\}(\boldsymbol{\omega})=\left(e^{i_{n} \boldsymbol{\omega} \cdot \boldsymbol{a}} \mathcal{F}\left\{f_{\text {odd }}\right\}(\boldsymbol{\omega})+e^{-i_{n} \boldsymbol{\omega} \cdot \boldsymbol{a}} \mathcal{F}\left\{f_{\text {even }}\right\}(\boldsymbol{\omega})\right) \mathcal{F}\{g\}(\boldsymbol{\omega}) .
$$

Proof. Using a similar argument as the proof of equation (24), we immediately get

$$
\begin{aligned}
\mathcal{F}\left\{\tau_{\boldsymbol{a}} f\right. & \star g\}(\boldsymbol{\omega}) \\
& =\mathcal{F}\left\{\tau_{\boldsymbol{a}} f\right\}(\boldsymbol{\omega}) \mathcal{F}\{g\}(\boldsymbol{\omega}) \\
& =\mathcal{F}\{f\}(\boldsymbol{\omega}) e^{-i_{n} \boldsymbol{\omega} \cdot \boldsymbol{a}} \mathcal{F}\{g\}(\boldsymbol{\omega}) \\
& =\left(e^{i_{n} \boldsymbol{\omega} \cdot \boldsymbol{a}} \mathcal{F}\left\{f_{\text {odd }}\right\}(\boldsymbol{\omega})+e^{-i_{n} \boldsymbol{\omega} \cdot \boldsymbol{a}} \mathcal{F}\left\{f_{\text {even }}\right\}(\boldsymbol{\omega})\right) \mathcal{F}\{g\}(\boldsymbol{\omega}),
\end{aligned}
$$

where, in the last line of (29), we have used the first equation in Lemma 3.3. CFT.

Now we establish the modulation property of the convolution theorem of the

Theorem 5.6. Let $f, g \in L^{2}\left(\mathbb{R}^{n} ; C l_{n, 0}\right)$. Then

$$
\begin{aligned}
\mathcal{F}\left\{f \star M_{\boldsymbol{\omega}_{0}} g\right\}(\boldsymbol{\omega})=\mathcal{F}\{f\}(-\boldsymbol{\omega}) \mathcal{F}\left\{g_{\text {odd }}\right\}(\boldsymbol{\omega} & \left.+\boldsymbol{\omega}_{0}\right) \\
& +\mathcal{F}\{f\}(\boldsymbol{\omega}) \mathcal{F}\left\{g_{\text {even }}\right\}\left(\boldsymbol{\omega}-\boldsymbol{\omega}_{0}\right),
\end{aligned}
$$

and

$$
\begin{aligned}
\mathcal{F}\left\{M_{\boldsymbol{\omega}_{0}} f \star g\right\}(\boldsymbol{\omega}) & =\left(\mathcal{F}\left\{f_{\text {odd }}\right\}\left(\boldsymbol{\omega}_{0}-\boldsymbol{\omega}\right)+\mathcal{F}\left\{f_{\text {even }}\right\}\left(-\boldsymbol{\omega}-\boldsymbol{\omega}_{0}\right)\right) \mathcal{F}\left\{g_{\text {odd }}\right\}(\boldsymbol{\omega}) \\
& +\left(\mathcal{F}\left\{f_{\text {odd }}\right\}\left(\boldsymbol{\omega}+\boldsymbol{\omega}_{0}\right)+\mathcal{F}\left\{f_{\text {even }}\right\}\left(\boldsymbol{\omega}-\boldsymbol{\omega}_{0}\right)\right) \mathcal{F}\left\{g_{\text {even }}\right\}(\boldsymbol{\omega}) .
\end{aligned}
$$


Proof. We only sketch the proof of (30), the other being similar. A direct computation gives

$$
\begin{aligned}
\mathcal{F}\{f & \left.\star M_{\boldsymbol{\omega}_{0}} g\right\}(\boldsymbol{\omega}) \\
& \stackrel{(14)}{=} \int_{\mathbb{R}^{n}} \int_{\mathbb{R}^{n}} f(\boldsymbol{y}) e^{i_{n} \boldsymbol{\omega}_{0} \cdot(\boldsymbol{x}-\boldsymbol{y})} g(\boldsymbol{x}-\boldsymbol{y}) d^{n} \boldsymbol{y} e^{-i_{n} \boldsymbol{\omega} \cdot \boldsymbol{x}} d^{n} \boldsymbol{x} \\
= & \int_{\mathbb{R}^{n}} f(\boldsymbol{y})\left[\int_{\mathbb{R}^{n}} e^{i_{n} \boldsymbol{\omega}_{0} \cdot(\boldsymbol{x}-\boldsymbol{y})} g(\boldsymbol{x}-\boldsymbol{y}) e^{-i_{n} \boldsymbol{\omega} \cdot \boldsymbol{x}} d^{n} \boldsymbol{x}\right] d^{n} \boldsymbol{y} \\
= & \int_{\mathbb{R}^{n}} f(\boldsymbol{y})\left[\int_{\mathbb{R}^{n}} e^{i_{n} \boldsymbol{\omega}_{0} \cdot \boldsymbol{z}} g(\boldsymbol{z}) e^{-i_{n} \boldsymbol{\omega} \cdot(\boldsymbol{y}+\boldsymbol{z})} d^{n} \boldsymbol{z}\right] d^{n} \boldsymbol{y} \\
= & \int_{\mathbb{R}^{n}} f(\boldsymbol{y})\left[\int_{\mathbb{R}^{n}} e^{i_{n} \boldsymbol{\omega}_{0} \cdot \boldsymbol{z}} g(\boldsymbol{z}) e^{-i_{n} \boldsymbol{\omega} \cdot \boldsymbol{z}} e^{-i_{n} \boldsymbol{\omega} \cdot \boldsymbol{y}} d^{n} \boldsymbol{z}\right] d^{n} \boldsymbol{y} \\
= & \int_{\mathbb{R}^{n}} f(\boldsymbol{y})\left[\int_{\mathbb{R}^{n}} e^{i_{n} \boldsymbol{\omega} \cdot \boldsymbol{y}} g_{\mathrm{odd}}(\boldsymbol{z}) e^{-i_{n}\left(\boldsymbol{\omega}+\boldsymbol{\omega}_{0}\right) \cdot \boldsymbol{z}} d^{n} \boldsymbol{z}\right. \\
& \left.+\int_{\mathbb{R}^{n}} e^{-i_{n} \boldsymbol{\omega} \cdot \boldsymbol{y}} g_{\mathrm{even}}(\boldsymbol{z}) e^{-i_{n}\left(\boldsymbol{\omega}-\boldsymbol{\omega}_{0}\right) \cdot \boldsymbol{z}} d^{n} \boldsymbol{z}\right] d^{n} \boldsymbol{y} \\
= & \int_{\mathbb{R}^{n}} f(\boldsymbol{y}) e^{i_{n} \boldsymbol{\omega} \cdot \boldsymbol{y}} d^{n} \boldsymbol{y} \mathcal{F}\left\{g_{\mathrm{odd}}\right\}\left(\boldsymbol{\omega}+\boldsymbol{\omega}_{0}\right)+\int_{\mathbb{R}^{n}} e^{-i_{n} \boldsymbol{\omega} \cdot \boldsymbol{y}} d^{n} \boldsymbol{y} \mathcal{F}\left\{g_{\mathrm{even}}\right\}\left(\boldsymbol{\omega}-\boldsymbol{\omega}_{0}\right) \\
= & \mathcal{F}\{f\}(-\boldsymbol{\omega}) \mathcal{F}\left\{g_{\mathrm{odd}}\right\}\left(\boldsymbol{\omega}+\boldsymbol{\omega}_{0}\right)+\mathcal{F}\{f\}(\boldsymbol{\omega}) \mathcal{F}\left\{g_{\mathrm{even}}\right\}\left(\boldsymbol{\omega}-\boldsymbol{\omega}_{0}\right),
\end{aligned}
$$

which was to be proved.

Remark 5.7. Note that if $g \in L^{2}\left(\mathbb{R}^{n} ; C l_{n, 0}\right)$ with $n=3(\bmod 4)$, then Theorem 5.6 has the form

$$
\mathcal{F}\left\{f \star M_{\boldsymbol{\omega}_{0}} g\right)=\mathcal{F}\{f\}(\boldsymbol{\omega}) \mathcal{F}\{g\}\left(\boldsymbol{\omega}-\boldsymbol{\omega}_{0}\right),
$$

which is of the same form as the modulation property of the convolution of the FT (see [11]).

We further establish the time-frequency shift of the convolution theorem of the CFT.

Theorem 5.8. Let $f, g \in L^{2}\left(\mathbb{R}^{n} ; C l_{n, 0}\right)$. Then

$$
\begin{aligned}
\mathcal{F}\left\{M_{\boldsymbol{\omega}_{0}} \tau_{\boldsymbol{a}} f \star g\right\}(\boldsymbol{\omega})=\left(e^{i_{n} \boldsymbol{\omega} \cdot \boldsymbol{a}} \mathcal{F}\left\{f_{\text {odd }}\right\}\left(\boldsymbol{\omega}_{0}-\boldsymbol{\omega}\right)\right. & \\
\left.+e^{-i_{n} \boldsymbol{\omega} \cdot \boldsymbol{a}} \mathcal{F}\left\{f_{\text {even }}\right\}\left(-\boldsymbol{\omega}-\boldsymbol{\omega}_{0}\right)\right) & \mathcal{F}\left\{g_{\text {odd }}\right\}(\boldsymbol{\omega})+\left(e^{i_{n} \boldsymbol{\omega} \cdot \boldsymbol{a}} \mathcal{F}\left\{f_{\text {odd }}\right\}\left(\boldsymbol{\omega}+\boldsymbol{\omega}_{0}\right)\right. \\
& \left.=+e^{-i_{n} \boldsymbol{\omega} \cdot \boldsymbol{a}} \mathcal{F}\left\{f_{\text {even }}\right\}\left(\boldsymbol{\omega}-\boldsymbol{\omega}_{0}\right)\right) \mathcal{F}\left\{g_{\text {odd }}\right\}(\boldsymbol{\omega}) .
\end{aligned}
$$

Proof. Applying equations (10) and (14), we immediately obtain

$$
\begin{aligned}
& \mathcal{F}\left\{M_{\boldsymbol{\omega}_{0}} \tau_{\boldsymbol{a}} f \star g\right\}(\boldsymbol{\omega}) \\
& =\int_{\mathbb{R}^{n}}\left(M_{\boldsymbol{\omega}_{0}} \tau_{\boldsymbol{a}} f \star g\right) e^{-i_{n} \boldsymbol{\omega} \cdot \boldsymbol{x}} d^{n} \boldsymbol{x} \\
& =\int_{\mathbb{R}^{n}} \int_{\mathbb{R}^{n}} e^{i_{n} \boldsymbol{\omega}_{0} \cdot \boldsymbol{y}} f(\boldsymbol{y}-\boldsymbol{a}) g(\boldsymbol{x}-\boldsymbol{y}) d^{n} \boldsymbol{y} e^{-i_{n} \boldsymbol{\omega} \cdot \boldsymbol{x}} d^{n} \boldsymbol{x}
\end{aligned}
$$




$$
\begin{aligned}
& =\int_{\mathbb{R}^{n}} e^{i_{n} \boldsymbol{\omega}_{0} \cdot \boldsymbol{y}} f(\boldsymbol{y}-\boldsymbol{a})\left[\int_{\mathbb{R}^{n}} g(\boldsymbol{x}-\boldsymbol{y}) e^{-i_{n} \boldsymbol{\omega} \cdot \boldsymbol{x}} d^{n} \boldsymbol{x}\right] d^{n} \boldsymbol{y} \\
& =\int_{\mathbb{R}^{n}} e^{i_{n} \boldsymbol{\omega}_{0} \cdot \boldsymbol{y}} f(\boldsymbol{y}-\boldsymbol{a})\left[\int_{\mathbb{R}^{n}} g(\boldsymbol{z}) e^{-i_{n} \boldsymbol{\omega} \cdot(\boldsymbol{y}+\boldsymbol{z})} d^{n} \boldsymbol{z}\right] d^{n} \boldsymbol{y} \\
& =\int_{\mathbb{R}^{n}} e^{i_{n} \boldsymbol{\omega}_{0} \cdot \boldsymbol{y}} f(\boldsymbol{y}-\boldsymbol{a})\left[\int_{\mathbb{R}^{n}} g(\boldsymbol{z}) e^{-i_{n} \boldsymbol{\omega} \cdot \boldsymbol{y}} e^{-i_{n} \boldsymbol{\omega} \cdot \boldsymbol{z}} d^{n} \boldsymbol{z}\right] d^{n} \boldsymbol{y} .
\end{aligned}
$$

We decompose $f$ and $g$ into $f_{\text {odd }}+f_{\text {even }}$ and $g_{\text {odd }}+g_{\text {even }}$, respectively. Then, we obtain

$$
\begin{aligned}
\mathcal{F}\{ & \left.M_{\boldsymbol{\omega}_{0}} \tau_{\boldsymbol{a}} f \star g\right\}(\boldsymbol{\omega}) \\
= & \int_{\mathbb{R}^{n}} e^{i_{n} \boldsymbol{\omega}_{0} \cdot \boldsymbol{y}} f(\boldsymbol{y}-\boldsymbol{a})\left[\int_{\mathbb{R}^{n}} e^{i_{n} \boldsymbol{\omega} \cdot \boldsymbol{y}} g_{\mathrm{odd}}(\boldsymbol{z}) e^{-i_{n} \boldsymbol{\omega} \cdot \boldsymbol{z}} d^{n} \boldsymbol{z}\right. \\
& \left.+\int_{\mathbb{R}^{n}} e^{-i_{n} \boldsymbol{\omega} \cdot \boldsymbol{y}} g_{\mathrm{even}}(\boldsymbol{z}) e^{-i_{n} \boldsymbol{\omega} \cdot \boldsymbol{z}} d^{n} \boldsymbol{z}\right] d^{n} \boldsymbol{y} \\
= & {\left[\int_{\mathbb{R}^{n}} f_{\mathrm{odd}}(\boldsymbol{y}-\boldsymbol{a}) e^{-i_{n}\left(\boldsymbol{\omega}_{0}-\boldsymbol{\omega}\right) \cdot \boldsymbol{y}} d^{n} \boldsymbol{y}\right.} \\
& \left.+\int_{\mathbb{R}^{n}} f_{\mathrm{even}}(\boldsymbol{y}-\boldsymbol{a}) e^{-i_{n}\left(-\boldsymbol{\omega}-\boldsymbol{\omega}_{0}\right) \cdot \boldsymbol{y}} d^{n} \boldsymbol{y}\right] \mathcal{F}\left\{g_{\mathrm{odd}}\right\}(\boldsymbol{\omega}) \\
& +\left[\int_{\mathbb{R}^{n}} f_{\text {odd }}(\boldsymbol{y}-\boldsymbol{a}) e^{-i_{n}\left(\boldsymbol{\omega}+\boldsymbol{\omega}_{0}\right) \cdot \boldsymbol{y}} d^{n} \boldsymbol{y}\right. \\
& \left.+\int_{\mathbb{R}^{n}} f_{\mathrm{even}}(\boldsymbol{y}-\boldsymbol{a}) e^{-i_{n}\left(\boldsymbol{\omega}-\boldsymbol{\omega}_{0}\right) \cdot \boldsymbol{y}} d^{n} \boldsymbol{y}\right] \mathcal{F}\left\{g_{\mathrm{even}}\right\}(\boldsymbol{\omega}) .
\end{aligned}
$$

which was to be proved.

Corollary 5.9. If $g \in L^{2}\left(\mathbb{R}^{n} ; C l_{n, 0}\right)$ with $n=3(\bmod 4)$. Then, Theorem 5.8 reduces to

$$
\begin{aligned}
\mathcal{F}\left\{M_{\boldsymbol{\omega}_{0}} \tau_{\boldsymbol{a}} f \star g\right\}(\boldsymbol{\omega})=\left(e^{i_{n}\left(\boldsymbol{\omega}_{0}-\boldsymbol{\omega}\right) \cdot \boldsymbol{a}} \mathcal{F}\left\{f_{\text {odd }}\right\}\left(\boldsymbol{\omega}+\boldsymbol{\omega}_{0}\right)\right. \\
\left.\quad+e^{-i_{n}\left(\boldsymbol{\omega}-\boldsymbol{\omega}_{0}\right) \cdot \boldsymbol{a}} \mathcal{F}\left\{f_{\text {even }}\right\}\left(\boldsymbol{\omega}-\boldsymbol{\omega}_{0}\right)\right) \mathcal{F}\{g\}(\boldsymbol{\omega}) .
\end{aligned}
$$

If $f \in L^{2}\left(\mathbb{R}^{n} ; C l_{n, 0}\right)$ with $n=3(\bmod 4)$, then

$$
\begin{aligned}
\mathcal{F}\left\{M_{\boldsymbol{\omega}_{0}} \tau_{\boldsymbol{a}} f \star g\right\}(\boldsymbol{\omega})=\left(e^{i_{n}\left(\boldsymbol{\omega}_{0}+\boldsymbol{\omega}\right) \cdot \boldsymbol{a}} \mathcal{F}\{f\}\left(-\boldsymbol{\omega}-\boldsymbol{\omega}_{0}\right) \mathcal{F}\left\{g_{\text {odd }}\right\}(\boldsymbol{\omega})\right. & \\
& \left.+e^{-i_{n}\left(\boldsymbol{\omega}-\boldsymbol{\omega}_{0}\right) \cdot \boldsymbol{a}} \mathcal{F}\{f\}\left(\boldsymbol{\omega}-\boldsymbol{\omega}_{0}\right)\right) \mathcal{F}\left\{g_{\text {even }}\right\}(\boldsymbol{\omega}) .
\end{aligned}
$$

Theorem 5.10. Let $f, g \in L^{2}\left(\mathbb{R}^{n} ; C l_{n, 0}\right)$. Then

$$
\begin{aligned}
\mathcal{F}\{f\}\left(-\boldsymbol{\omega}-\boldsymbol{\omega}_{0}\right) e^{i_{n}\left(\boldsymbol{\omega}-\boldsymbol{\omega}_{0}\right) \cdot \boldsymbol{a}} \mathcal{F}\left\{g_{\text {odd }}\right\} & (\boldsymbol{\omega}) \\
& +\mathcal{F}\{f\}\left(\boldsymbol{\omega}-\boldsymbol{\omega}_{0}\right) e^{-i_{n}\left(\boldsymbol{\omega}+\boldsymbol{\omega}_{0}\right) \cdot \boldsymbol{a}} \mathcal{F}\left\{g_{\text {even }}\right\}(\boldsymbol{\omega}) .
\end{aligned}
$$

Proof. By the definition of the CFT (14) and Clifford convolution (17), we have

$$
\mathcal{F}\left\{f \star \tau_{\boldsymbol{a}} M_{\boldsymbol{\omega}_{0}} g\right\}(\boldsymbol{\omega})
$$




$$
\begin{aligned}
\stackrel{(14)}{=} & \int_{\mathbb{R}^{n}} \int_{\mathbb{R}^{n}} f(\boldsymbol{y}) e^{i_{n} \boldsymbol{\omega}_{0} \cdot(\boldsymbol{y}-\boldsymbol{a})} g(\boldsymbol{x}-\boldsymbol{y}-\boldsymbol{a}) d^{n} \boldsymbol{y} e^{-i_{n} \boldsymbol{\omega} \cdot \boldsymbol{x}} d^{n} \boldsymbol{x} \\
= & \int_{\mathbb{R}^{n}} f(\boldsymbol{y}) e^{i_{n} \boldsymbol{\omega}_{0} \cdot(\boldsymbol{y}-\boldsymbol{a})}\left[\int_{\mathbb{R}^{n}} g(\boldsymbol{x}-\boldsymbol{y}-\boldsymbol{a}) e^{-i_{n} \boldsymbol{\omega} \cdot \boldsymbol{x}} d^{n} \boldsymbol{x}\right] d^{n} \boldsymbol{y} \\
= & \int_{\mathbb{R}^{n}} f(\boldsymbol{y}) e^{i_{n} \boldsymbol{\omega}_{0} \cdot(\boldsymbol{y}-\boldsymbol{a})}\left[\int_{\mathbb{R}^{n}} g(\boldsymbol{z}-\boldsymbol{a}) e^{-i_{n} \boldsymbol{\omega} \cdot(\boldsymbol{y}+\boldsymbol{z})} d^{n} \boldsymbol{z}\right] d^{n} \boldsymbol{y} \\
= & \int_{\mathbb{R}^{n}} f(\boldsymbol{y}) e^{i_{n} \boldsymbol{\omega}_{0} \cdot(\boldsymbol{y}-\boldsymbol{a})}\left[\int_{\mathbb{R}^{n}} g(\boldsymbol{z}-\boldsymbol{a}) e^{-i_{n} \boldsymbol{\omega} \cdot \boldsymbol{y}} e^{-i_{n} \boldsymbol{\omega} \cdot \boldsymbol{z}} d^{n} \boldsymbol{z}\right] d^{n} \boldsymbol{y} \\
= & \int_{\mathbb{R}^{n}} f(\boldsymbol{y}) e^{i_{n} \boldsymbol{\omega}_{0} \cdot(\boldsymbol{y}-\boldsymbol{a})}\left[\int_{\mathbb{R}^{n}} e^{i_{n} \boldsymbol{\omega} \cdot \boldsymbol{y}} g_{\mathrm{odd}}(\boldsymbol{z}-\boldsymbol{a}) e^{-i_{n} \boldsymbol{\omega} \cdot \boldsymbol{z}} d^{n} \boldsymbol{z}\right. \\
& \left.+\int_{\mathbb{R}^{n}} e^{-i_{n} \boldsymbol{\omega} \cdot \boldsymbol{y}} g_{\mathrm{even}}(\boldsymbol{z}-\boldsymbol{a}) e^{-i_{n} \boldsymbol{\omega} \cdot \boldsymbol{z}} d^{n} \boldsymbol{z}\right] d^{n} \boldsymbol{y} \\
= & \int_{\mathbb{R}^{n}} f(\boldsymbol{y}) e^{-i_{n}\left(-\boldsymbol{\omega}-\boldsymbol{\omega}_{0}\right) \cdot \boldsymbol{y}} d^{n} \boldsymbol{y} e^{i_{n}\left(\boldsymbol{\omega}-\boldsymbol{\omega}_{0}\right) \cdot \boldsymbol{a}} \mathcal{F}\left\{g_{\mathrm{odd}}\right\}(\boldsymbol{\omega}) \\
& +\int_{\mathbb{R}^{n}} f(\boldsymbol{y}) e^{-i_{n}\left(\boldsymbol{\omega}-\boldsymbol{\omega}_{0}\right) \cdot \boldsymbol{y}} d^{n} \boldsymbol{y} e^{-i_{n}\left(\boldsymbol{\omega}+\boldsymbol{\omega}_{0}\right) \cdot \boldsymbol{a}} \mathcal{F}\left\{g_{\mathrm{even}}\right\}(\boldsymbol{\omega}) \\
= & \mathcal{F}\{f\}\left(-\boldsymbol{\omega}-\boldsymbol{\omega}_{0}\right) e^{i_{n}\left(\boldsymbol{\omega}-\boldsymbol{\omega}_{0}\right) \cdot \boldsymbol{a}} \mathcal{F}\left\{g_{\mathrm{odd}}\right\}(\boldsymbol{\omega}) \\
& +\mathcal{F}\{f\}\left(\boldsymbol{\omega}-\boldsymbol{\omega}_{0}\right) e^{-i_{n}\left(\boldsymbol{\omega}+\boldsymbol{\omega}_{0}\right) \cdot \boldsymbol{a}} \mathcal{F}\left\{g_{\mathrm{even}}\right\}(\boldsymbol{\omega}),
\end{aligned}
$$

which was to be proved.

Corollary 5.11. It is straightforward to check that for $g \in L^{2}\left(\mathbb{R}^{n} ; C l_{n, 0}\right)$ with $n=3(\bmod 4)$, Theorem 5.8 reduces to

$$
\mathcal{F}\left\{f \star \tau_{\boldsymbol{a}} M_{\boldsymbol{\omega}_{0}} g\right\}(\boldsymbol{\omega})=\mathcal{F}\{f\}\left(\boldsymbol{\omega}-\boldsymbol{\omega}_{0}\right) e^{-i_{n}\left(\boldsymbol{\omega}+\boldsymbol{\omega}_{0}\right) \cdot \boldsymbol{a}} \mathcal{F}\{g\}(\boldsymbol{\omega}) .
$$

By applying the inverse CFT we get the following result, which is very important for solving partial differential equations in Clifford algebra.

Theorem 5.12. Let $f, g \in L^{2}\left(\mathbb{R}^{n} ; C l_{n, 0}\right)$. Then

$$
\mathcal{F}^{-1}[\mathcal{F}\{f\}(\boldsymbol{\omega}) \mathcal{F}\{g\}(\boldsymbol{\omega})](\boldsymbol{x})=\left(f \star g_{\text {odd }}\right)(-\boldsymbol{x})+\left(f \star g_{\text {even }}\right)(\boldsymbol{x})
$$

Or, equivalently,

$$
\mathcal{F}\{f\}(\boldsymbol{\omega}) \mathcal{F}\{g\}(\boldsymbol{\omega})=\mathcal{F}\left\{\left(f \star g_{\text {odd }}\right)(-\cdot)\right\}(\boldsymbol{\omega})+\mathcal{F}\left\{f \star g_{\text {even }}\right\}(\boldsymbol{\omega})
$$


Proof. According to the inverse CFT, the left hand-side of (34) leads to

$$
\begin{aligned}
\mathcal{F}^{-1}[ & \mathcal{F}\{f\}(\boldsymbol{\omega}) \mathcal{F}\{g\}(\boldsymbol{\omega})](\boldsymbol{x}) \\
\stackrel{(16)}{=} & \frac{1}{(2 \pi)^{n}} \int_{\mathbb{R}^{n}} \int_{\mathbb{R}^{n}} \mathcal{F}\{f\}(\boldsymbol{\omega}) g(\boldsymbol{y}) e^{-i_{n} \boldsymbol{\omega} \cdot \boldsymbol{y}} d^{n} \boldsymbol{y} e^{i_{n} \boldsymbol{\omega} \cdot \boldsymbol{x}} d^{n} \boldsymbol{\omega} \\
= & \frac{1}{(2 \pi)^{n}} \int_{\mathbb{R}^{n}} \int_{\mathbb{R}^{n}} \mathcal{F}\{f\}(\boldsymbol{\omega})\left(g_{\text {odd }}(\boldsymbol{y})+g_{\text {even }}(\boldsymbol{y})\right) e^{i_{n} \boldsymbol{\omega} \cdot(\boldsymbol{x}-\boldsymbol{y})} d^{n} \boldsymbol{\omega} d^{n} \boldsymbol{y} \\
= & \frac{1}{(2 \pi)^{n}} \int_{\mathbb{R}^{n}} \int_{\mathbb{R}^{n}} \mathcal{F}\{f\}(\boldsymbol{\omega}) e^{i_{n} \boldsymbol{\omega} \cdot(\boldsymbol{y}-\boldsymbol{x})} d^{n} \boldsymbol{\omega} g_{\text {odd }}(\boldsymbol{y}) d^{n} \boldsymbol{y} \\
& +\frac{1}{(2 \pi)^{n}} \int_{\mathbb{R}^{n}} \int_{\mathbb{R}^{n}} \mathcal{F}\{f\}(\boldsymbol{\omega}) e^{i_{n} \boldsymbol{\omega} \cdot(\boldsymbol{x}-\boldsymbol{y})} d^{n} \boldsymbol{\omega} g_{\text {even }}(\boldsymbol{y}) d^{n} \boldsymbol{y} \\
= & \int_{\mathbb{R}^{n}} f(\boldsymbol{y}-\boldsymbol{x}) g_{\text {odd }}(\boldsymbol{y}) d^{n} \boldsymbol{y}+\int_{\mathbb{R}^{n}} f(\boldsymbol{x}-\boldsymbol{y}) g_{\text {even }}(\boldsymbol{y}) d^{n} \boldsymbol{y} \\
& \stackrel{(18)}{=}\left(f \star g_{\text {odd }}\right)(-\boldsymbol{x})+\left(f \star g_{\text {even }}\right)(\boldsymbol{x}),
\end{aligned}
$$

which finishes the proof.

The following corollary is a special case of Theorem 5.12.

Corollary 5.13. Let $f \in L^{2}\left(\mathbb{R}^{n} ; C l_{n, 0}\right)$. If we assume that $g \in L^{2}\left(\mathbb{R}^{n} ; C l_{n, 0}\right)$ for $n=3(\bmod 4)$, then

$$
\mathcal{F}\{f\}(\boldsymbol{\omega}) \mathcal{F}\{g\}(\boldsymbol{\omega})=\mathcal{F}\{f \star g\}(\boldsymbol{\omega}) .
$$

Remark 5.14. According to (2), formula (35) also holds if only if $g \in L^{2}\left(\mathbb{R}^{n} ; C l_{n, 0}\right)$ is an even grade multivector.

Acknowledgements. The authors would like to thank the anonymous reviewer for valuable comments and suggestions which improved the clarity and quality of this paper. The first author is partially supported by Hibah Penelitian Kompetisi Internal tahun 2013 (No. 110/UN4-.42/LK.26/SP-UH/2013) from the Hasanuddin University, Indonesia. The second author is partially supported by JSPS.KAKENHI (C)25400202 of Japan and the third author is partially supported by NSERC of Canada.

\section{REFERENCES}

[1] Mawardi, B. and Hitzer, E., Clifford Fourier transformation and uncertainty principle for the Clifford geometric algebra $\mathrm{Cl}_{3,0}$, Adv. Appl. Clifford Algebr., 16(1) (2006), 41-61.

[2] Mawardi, B., Adji, S. and Zhao, J., Clifford algebra-valued wavelet transform on multivector fields, Adv. Appl. Clifford Algebr., 21(1) (2011), 13-30.

[3] Hitzer, E., Quaternion Fourier transform on quaternion fields and generalizations, Adv. Appl. Clifford Algebr., 17(3) (2007), 497-517.

[4] Hitzer, E. and Mawardi, B., Clifford Fourier transform on multivector fields and uncertainty principle for dimensions $n=2(\bmod 4)$ and $n=3(\bmod 4)$, Adv. Appl. Clifford Algebr., 18(3-4) (2008), 715-736. 
[5] Brackx, F., Delanghe, R. and Sommen, F., Clifford Analysis, Research Notes in Mathematics, 76. Pitman, Boston, MA, 1982.

[6] Sommen, F., A product and an exponential function in hypercomplex function theory, $A p$ plicable Anal., 12(1) (1981), 13-26.

[7] De Bie, H., De Schepper, N. and Sommen, F., The class of Clifford-Fourier transforms, J. Fourier Anal. Appl., 17(6) (2011), 1198-1231.

[8] De Bie, H. and De Schepper, N., The fractional Clifford-Fourier transforms, Complex Anal. Oper. Theory, 6(5) (2012), 1047-1067.

[9] Ebling, J. and Scheuermann, G., Clifford Fourier transform on vector fields, IEEE Transactions on Visualization and Computer Graphics, 11(4) (2005), 469-479.

[10] Bahri, M., Clifford windowed Fourier transform applied to linear time-varying systems, Appl. Math. Sci. (Ruse), 6(58) (2012), 2857-2864.

[11] Bracewell, R. N., The Fourier Transform and its Applications, McGraw-Hill International Editions, Singapore, 2000

[12] Bujack, R., Scheuermann, G. and Hitzer, E., A general geometric Fourier transform convolution theorem, Adv. Appl. Clifford Algebr., 23(1) (2013), 15-38.

[13] Sangwine, S. J., Color images edge detector based on quaternion convolution, Electronics Letters, 34(10) (1998), 969-971.

[14] Batard, T., Berthier, M. and Saint-Jean, C., Clifford-Fourier transform for color image processing, In: Geometric Algebra Computing in Engineering and Computer Science, pp. 135162, Springer London, 2010.

[15] Fu, Y., Kähler, U. and Cerejeiras, P., The Balian-Low theorem for the windowed quaternionic Fourier transform, Adv. Appl. Clifford Algebr., 22(4) (2012), 1025-1040. 